\title{
PHYLOGROUP PROFILING AND THE GENETIC DETERMINANTS OF EXTENDED SPECTRUM BETA-LACTAMASE PRODUCING Escherichia coli ISOLATED FROM CLINICAL SAMPLES IN LAGOS, NIGERIA
}

\author{
Olalekan, A. O. ${ }^{1} *$, Onwugamba, F. ${ }^{2}$, Iwalokun, B. ${ }^{3}$ \\ ${ }^{1}$ Department of Medical Laboratory Science, College of Medicine, University of Lagos, Nigeria. ${ }^{2}$ Institute of Medical \\ Microbiology, University Hospital Münster, Münster, Germany, (E-mail: onwugamba.francis@gmail.com). \\ ${ }^{3}$ Molecular Biology and Biotechnology Department, Nigerian Institute of Medical Research, Lagos, Nigeria, (E-mail: \\ biiwalokun@gmail.com). \\ *Corresponding author's e-mail: adesolaolalekan@unilag.edu.ng, Tel.: +2347084104478.
}

(Received: $3^{\text {rd }}$ August, 2020; Accepted: $12^{\text {th }}$ November, 2020)

\section{ABSTRACT}

\begin{abstract}
Escherichia coli (E. coli) is one of the most commonly isolated multi-drug resistant bacteria (MDR) responsible for a wide spectrum of human infections characterized by virulence variation and capacity to produce extendedspectrum beta-lactamase (ESBL). However, the phylo-groups that most of the MDR E. coli strains belong are unknown. Here, the distribution of gene determinants of ESBL producing E. colistrains from clinical samples in Lagos, Nigeria in relation to their phylogroups was studied. One hundred and thirteen ESBL- producing E. coli isolates were prospectively collected from infection sites namely wound infection $(n=39)$, urinary tract infection $(n=57)$, puerperal sepsis $(n=6)$, sepsis $(n=5)$, enteritis $(n=3)$, and throat infection $(n=3)$ from four specialist and tertiary hospitals in Lagos State, Nigeria. The isolates were confirmed with MALDI-TOF mass spectrometry (Microflex LT, Bruker Daltonics, Germany). Antimicrobial susceptibility testing was carried out with the VITEK 2 system card N- 232 (bioMerieux SA, France), and the interpretation guidelines of the European Committee on Antimicrobial Susceptibility Testing (EUCAST) were adopted. The E. coli phylogroups were classified according to the Clermont typing scheme, while ESBL genes $\left(b l a_{S H V}\right.$, bla $a_{T E M}$, and $\left.b l a_{C T X-M}\right)$ were detected by Multiplex PCR amplification. The most predominant phylogroup was B2 65 (58.0\%), followed by Clades I \& II 16 (14.1\%), E 12 (10.6\%), F 6 (5.3\%), A 3 (2.7\%), C 2 (1.8\%), and B1 1 (0.9\%), while 8 (7.1\%) strains were unclassifiable. Out of all the phylogroups, strains belonging to group B2 harboured $b l a_{C T X-M} 10(15.4 \%)$, one $b l a_{T E M}$ $(1.5 \%)$ and $54(83 \%)$ of co-existing $\left(b l a_{C T X-M}\right.$ and $\left.b l_{T E M}\right) ; 2(100 \%)$ phylogroup $C$ harboured co-existing genes $\left(b l a_{C T X-M}\right.$ and $\left.b l a_{T E M}\right)$ and the only isolate with phylogroup B1 harboured co-existing gene $\left(b l a_{C T X-M}\right.$ and $\left.b l a_{T E M}\right)$. Our results show a high prevalence of bla $a_{C T X-M+} b l a_{T E M} c 0$ - existing among ESBL-E. coli strains belonging to phylogroup $\mathrm{B} 2$ in the studied healthcare settings. Strategies for continuous monitoring, prevention, and control of ESBLproducing E. coli strains to checkmate their nosocomial transmission are urgently needed in the studied hospitals.
\end{abstract}

Keywords: E. coli, ESBL, Phylogroup, bla $a_{\mathrm{CTX}-\mathrm{M},}$ and $b l a_{\mathrm{TEM}}$

\section{INTRODUCTION}

Extended spectrum beta-lactamase (ESBL) producing Escherichia coli (E. coli) infections is a public health threat because these infections are difficult to manage due to multiple antibiotic resistance, leading to the availability of only a few antibiotics, that are effective against the infections. E. coli strains are normal flora of the gastrointestinal tract but can also cause serious infections in humans ranging from urinary tract infections (Onanuga et al., 2019), surgical site infections to sepsis globally (Adenipekun et al., 2016; Nwafia et al., 2019a). This situation is compounded by their resistance to the third generation cephalosporins which are commonly used to treat associated infections (Umeokonkwo et al., 2019). The resultant effects of this multidrug resistance include increased cost of management of various infections and prolonged hospital stay in many cases (Lakoh et al., 2020). The common resistance genes associated with ESBL production and multi-drug resistance in $E$. coli are plasmid-borne and are designated as $b l a_{S H V}$, $b l a_{T E M}$, and $b l a_{C T X-M}$. Carriage of these genes by ESBL producing E. coli at varying prevalence rates in different settings have previously been reported in Nigeria (Raji et al., 2013; Hertz et al., 2019; Nwafia et al., 2019a), albeit their phylotypes have not been elucidated.

Moreover, E. coli strains have a clonal structure in their populations that has been classified by scientists into phylotypes (Clermont et al., 2013; Munkhdelger et al., 2017) and these phylotypes are associated with virulence such as the phylogroup B2 and D responsible for extra-intestinal 
infections in humans (Clermont et al., 2013) and the commensals belong to phylogroups B1 and A (Duriez et al., 2001). Also, phylogroup C is close to B1, many of the formerly assigned phylogroup D strains are now re-assigned as phylogroup $\mathrm{E}$, and $\mathrm{F}$ is a sister to B2 (Clermont et al., 2013). This information is pertinent for classifying the resistant strains in our isolate collection into the global epidemiological phylogroup context to know the resistance gene determinants that are associated with a particular phylogroup. Here, we used the revisited Clermont Escherichia coli phylotyping method to categorize the strains in this study (Clermont et al., 2013).

Notably, multidrug-resistant infections have caused enormous problems globally especially in low and middle-income countries with a lack of functional antibiotic stewardship, indiscriminate use of antibiotics, and lack of control of antibiotics use in veterinary and agriculture contributing to the high resistance experienced due to antibiotic selection pressure. Therefore, an understanding of the transmission of ESBL genes in relations to phylogroup will further provide new knowledge on the molecular epidemiology of multi-drug resistant E. coli and their virulent disposition, since the information about the ESBL genes and their phylogroups among the strains isolated from the clinical specimen in Nigeria is scarce, Here, we studied the distribution of gene determinants and phylogroup profiles of clinical ESBL-producing E. coli strain from four health facilities in Lagos State, Nigeria.

\section{MATERIALS AND METHODS}

Isolate Collection, Identification, and Antimicrobial Susceptibility Testing

One hundred and thirteen isolates of ESBLproducing E. coli from our previous study were used for this study (Olalekan et al., 2020). The isolates were collected from various infection sites in humans namely wound infection $(n=39)$, urinary tract infection $(\mathrm{n}=57)$, puerperal sepsis $(n=6)$, sepsis $(n=5)$, enteritis $(n=3)$, and throat infection $(\mathrm{n}=3)$ at the Hospital Laboratory of National Orthopaedic Hospital Igbobi (33); Lagos State University Teaching Hospital Ikeja (8); Lagos University Teaching Hospital Idi-araba (67); Federal Neuro-psychiatric Hospital Yaba (3), all in Lagos State, southwest Nigeria. The isolates were collected between July 2016 to January 2018.

Identification of the bacterial strains was done conventionally using the indole production test, citrate utilization test, and lactose fermentation properties of the strains. The isolates were later confirmed with MALDI-TOF mass spectrometry (Microflex LT, Bruker Daltonics, Germany).

The antimicrobial susceptibility profile of the isolates was carried out with the VITEK 2 system card N- 232 (bioMerieux SA, France), and the interpretation guidelines of the European Committee on Antimicrobial Susceptibility Testing (EUCAST version 10.0) were adopted with the use of the clinical breakpoints (https://eucast.org/clinical_breakpoints/). The ESBL status was confirmed by the VITEK 2 system.

Ethical approval was obtained from the Health Research Ethics Committee of the College of Medicine of the University of Lagos (CMUL/HREC/05/17/136).

\section{Molecular Characterization of the ESBL Gene Determinants}

DNA extraction was performed using the QIAamp DNA Mini Kit (Qiagen, Germany) according to the manufacturer's instructions by adding $30 \mu \mathrm{l}$ proteinase $\mathrm{K}$ and $180 \mu \mathrm{l}$ of ATL buffer to the emulsified discrete colony and incubated at $56{ }^{\circ} \mathrm{C}$ at $900 \mathrm{rpm}$; other DNA extraction procedures were according to the manufacturer's guidelines. This is followed by amplification of the ESBL genes (bla $a_{S H V}, b l a_{\text {TEM }}$, and $\left.b l a_{C T X: M}\right)$ using a multiplex PCR protocol in a 25 $\mu l$ reaction containing $1.9 \mu \mathrm{l}$ of each pair of primer, $12.5 \mu$ l of Red-Taq, $1 \mu$ l of DNA, and 0.1 $\mu$ of PCR grade water. The amplification conditions were set at the initial denaturation step of $95^{\circ} \mathrm{C}$ for 5 minutes, 30 cycles of denaturation at $94^{\circ} \mathrm{C}$ for 30 seconds, annealing at $60^{\circ} \mathrm{C}$ for 30 seconds, extension at $72^{\circ} \mathrm{C}$ for 2 minutes, and one final extension step at $72{ }^{\circ} \mathrm{C}$ for 10 minutes. The sequences of the primers were bla-SHV.SE 5'atgcgttatattcgcctgtg 3'; bla-SHV.AS 5'tgctttgttattcgggccaa3'; TEM-164.SE 5'tcgccgcatacactattctcagaatga3'; TEM-165.AS 5'acgctcaccggetccagatttat $3^{\prime} ;$ CTX-M-U1 
5'atgtgcagyaccagtaargtkatggc3'; CTX-M-U2 5'tgggtraartargtsaccagaaycagcgg3' (Monstein et al., 2007). Amplicon size was 747 bp, 445 bp, and 593 bp respectively, and amplicons were separated on a $1.8 \%$ agarose gel.

\section{Phylotyping}

The E. coli isolates in this study were classified into phylotypes using the revisited Clermont typing scheme as published (Clermont et al., 2013) targeting the following genes chu $A$, yja $A$, TspE4.C2, $\operatorname{arp} A$, and $\operatorname{trp} A$ using the following corresponding primers as follows chuA $1 \& 2$, (279bp), yjaA1\&2 TspE4C2.1\&2 (211bp), TspE4.C2 (152bp), ArpAgpE_F\&R (301bp), trpAgpC.1\&.2 (219bp) as published and the amplification products were analyzed on 3\% agarose gel. The phylogroup assignment was carried out according to the laydown scheme by Clermont et al.(2013) and the interpretation is indicated in table 1.

\section{Data Analysis}

All the characteristics of the strains such as infection type, type of sample, gender, and age of patients with corresponding isolates, antimicrobial susceptibility profiles, resistance genes, and the phylogroups were entered into the
SPSS version 20 statistical package and descriptive analysis was performed to determine the frequencies.

\section{RESULTS}

A total of one hundred and thirteen isolates of $E$. coli from clinical specimens were studied. Out of which $56(49.6 \%)$ were recovered from male and $57(50.4 \%)$ from female patients. The age range was from 4 days to 79 years old with a mean age of 43 years. The type of samples was as follows: urine $57(50.4 \%)$, wound swabs 39 (34.5\%), high vaginal swab $6(5.3 \%)$, blood culture $5(4.4 \%)$, stool 3 $(2.7 \%)$, and sputum $3(2.7 \%)$.

The antimicrobial-resistant profiles of the ESBLproducing E. coli strain in this study show that all isolates were resistant to piperacillin, cefotaxime, and moxifloxacin, The order of resistance to the remaining antibiotics is ceftazidime 111 (98\%), aztreonam 111 (98\%), cefepime 110 (97.4\%), ciprofloxacin $109(96.5 \%)$, trimethoprim /sulphamethoxazole $106(94 \%)$, tobramycin 91 (80.5\%), gentamicin 88 (80\%), $46(41 \%)$ to piperacillin/tazobactam, meropenem $13(11.5 \%)$, imipenem 11 (9.7\%) and amikacin 11 (9.7\%). All the isolates were however $100 \%$ susceptible to tigecycline and colistin as presented in table 2 .

Table 1: Number and Percentage of Antibiotic Resistant Strains and their Various Phylo-groups

\begin{tabular}{|c|c|c|c|c|c|c|c|c|c|}
\hline Antibiotic resistance N (\%) & $\mathbf{A ( 3 )}$ & B1 (1) & B2 (65) & $\mathrm{C} \mathrm{(2)}$ & E (12) & F (6) & $\begin{array}{l}\text { Clade I or } \\
\text { II (16) }\end{array}$ & $\begin{array}{l}\text { unclassifiable } \\
\text { (8) }\end{array}$ & $\begin{array}{l}\text { Total } \\
\text { (113) }\end{array}$ \\
\hline Piperacillin/Tazobactam & $3(100 \%)$ & 0 & $16(25 \%)$ & $2(100 \%)$ & $6(50 \%)$ & $2(33 \%)$ & $13(81 \%)$ & $4(50 \%)$ & $46(41 \%)$ \\
\hline Cefotaxime & $3(100 \%)$ & $1(100 \%)$ & $65(100 \%)$ & $2(100 \%)$ & $12(100 \%)$ & $6(100 \%)$ & $16(100 \%)$ & $8(100 \%)$ & $113(100 \%)$ \\
\hline Ceftazidime & $3(100 \%)$ & $1(100 \%)$ & $64(98 \%)$ & $2(100 \%)$ & $12(100 \%)$ & $5(83 \%)$ & $16(100 \%)$ & $8(100 \%)$ & $111(98 \%)$ \\
\hline Cefepime & $3(100 \%)$ & $1(100 \%)$ & $64(98 \%)$ & $2(100 \%)$ & $12(100 \%)$ & $4(67 \%)$ & $16(100 \%)$ & $8(100 \%)$ & $110(97 \%)$ \\
\hline Aztreonam & $3(100 \%)$ & $1(100 \%)$ & $65(100 \%)$ & $2(100 \%)$ & $11(92 \%)$ & $5(83 \%)$ & $16(100 \%)$ & $8(100 \%)$ & $111(98 \%)$ \\
\hline Amikacin & $1(33.3 \%)$ & 0 & $4(6 \%)$ & $2(100 \%)$ & $2(17 \%)$ & $2(33 \%)$ & $5(31 \%)$ & 0 & $16(14 \%)$ \\
\hline Gentamicin & $1(33.3 \%)$ & 0 & $55(85 \%)$ & $2(100 \%)$ & $6(50 \%)$ & $5(83 \%)$ & $13(81 \%)$ & $6(75 \%)$ & $88(78 \%)$ \\
\hline Tobramycin & $3(100 \%)$ & 0 & $55(85 \%)$ & $2(100 \%)$ & $7(58 \%)$ & $5(83 \%)$ & $13(81 \%)$ & $6(75 \%)$ & $91(81 \%)$ \\
\hline Ciprofloxacin & $3(100 \%)$ & $1(100 \%)$ & $65(100 \%)$ & $2(100 \%)$ & $11(92 \%)$ & $6(100 \%)$ & $15(94 \%)$ & $6(75 \%)$ & $109(97 \%)$ \\
\hline Moxifloxacin & $3(100 \%)$ & $1(100 \%)$ & $65(100 \%)$ & $2(100 \%)$ & $12(100 \%)$ & $6(100 \%)$ & $16(100 \%)$ & $6(75 \%)$ & $109(97 \%)$ \\
\hline Tigecycline & 0 & 0 & 0 & 0 & 0 & 0 & 0 & 0 & 0 \\
\hline Trimethoprim/Sulphamethoxazole & $3(100 \%)$ & $1(100 \%)$ & $62(95 \%)$ & $2(100 \%)$ & $11(92 \%)$ & $6(100 \%)$ & $15(94 \%)$ & $6(75 \%)$ & $106(94 \%)$ \\
\hline
\end{tabular}


Table 2: Interpretation of the Phylogroup Profiles Using the Modified Extended Quadruplex Phylotyping Method

\begin{tabular}{llllll}
\hline Phylogroups & $\begin{array}{l}\text { chuA } \\
(279 b p)\end{array}$ & $\begin{array}{l}\text { yjaA } \\
(211 b p)\end{array}$ & $\begin{array}{l}\text { TspE4:C2 } \\
(152 b p)\end{array}$ & $\begin{array}{l}\text { ArpAgpE } \\
(301 b p)\end{array}$ & $\begin{array}{l}\text { trpAgpC } \\
(219 b p)\end{array}$ \\
\hline A & - & - & - & + & - \\
B1 & - & - & + & + & - \\
B2 & + & + & - & - & - \\
B2 & + & + & + & - & - \\
C & - & + & - & + & + \\
E & + & - & + & + & - \\
F & + & - & - & - & - \\
Clade 1 or 11 & - & + & - & - & - \\
\hline
\end{tabular}

Key, $+=$ Presence of the gene, $-=$ Absence of the gene

Overall, high rates of resistance were spread across all the phylogroups as shown in table 2 . None of the strains in this study was resistant to tigecycline and colistin. Only $9.7 \%$ of the isolates from all the phylogroups were resistant to amikacin and the only two strains that belonged to phylogroup $C$ were notably resistant to amikacin.

The distribution of the resistance genes in relation to the phylogroups is presented in figure 1 . The most predominant phylogroup is B2 $(65=58 \%)$, followed by Clades I\&II 16 (14\%), E 12 (11\%), F 6 (5\%), A $3(2.7 \%)$, C $2(1.8 \%)$ and B1 (0.9\%). Eight $(7.1 \%)$ strain phylogroups were unclassifiable.
Out of the one hundred and thirteen strains of $E$. coli in this study, 110 (97.3\%) harboured the antimicrobial-resistant gene either singly or in combination which includes $b l a_{\text {СTX-M }} 21$ (18.6\%), $b l a_{\text {TEM }} 1(0.9 \%), b l a_{C T X-M}$ and $b l a_{T E M}$ combination was $88(77.9 \%)$, none of the strains harbour bla $a_{S H V}$ in this study. Phylogroup B2 harboured majority of the classes of the resistance genes, in single as $b l a_{\text {CTX-M }}(10=15.4 \%), b l a_{\text {TEM }}(1=1.5 \%)$ and in combination as bla $a_{C T X M}$ and $b l a_{\text {TEM }}(54=83 \%)$. Phylogroup C harboured co-existing genes $\left(b l a_{C T X}\right.$. ${ }_{M}$ and $\left.b l a_{T E M}\right)=2(100 \%)$ and phylogroup B1 $\left(b l a_{C T X}\right.$. ${ }_{M}$ and $\left.b l a_{T E M}\right)=1(100 \%)$.

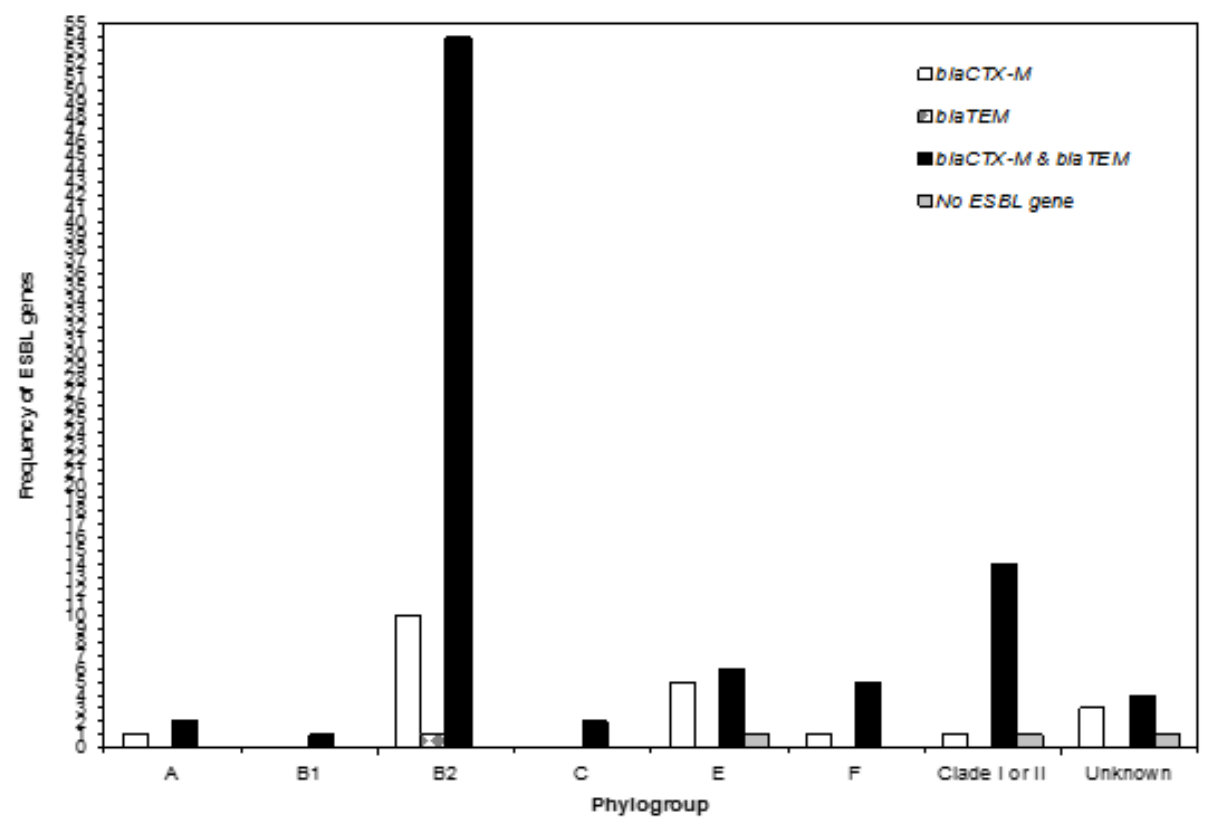

Figure 1. Distribution of ESBL Genes and their Associated Phylogroups. 


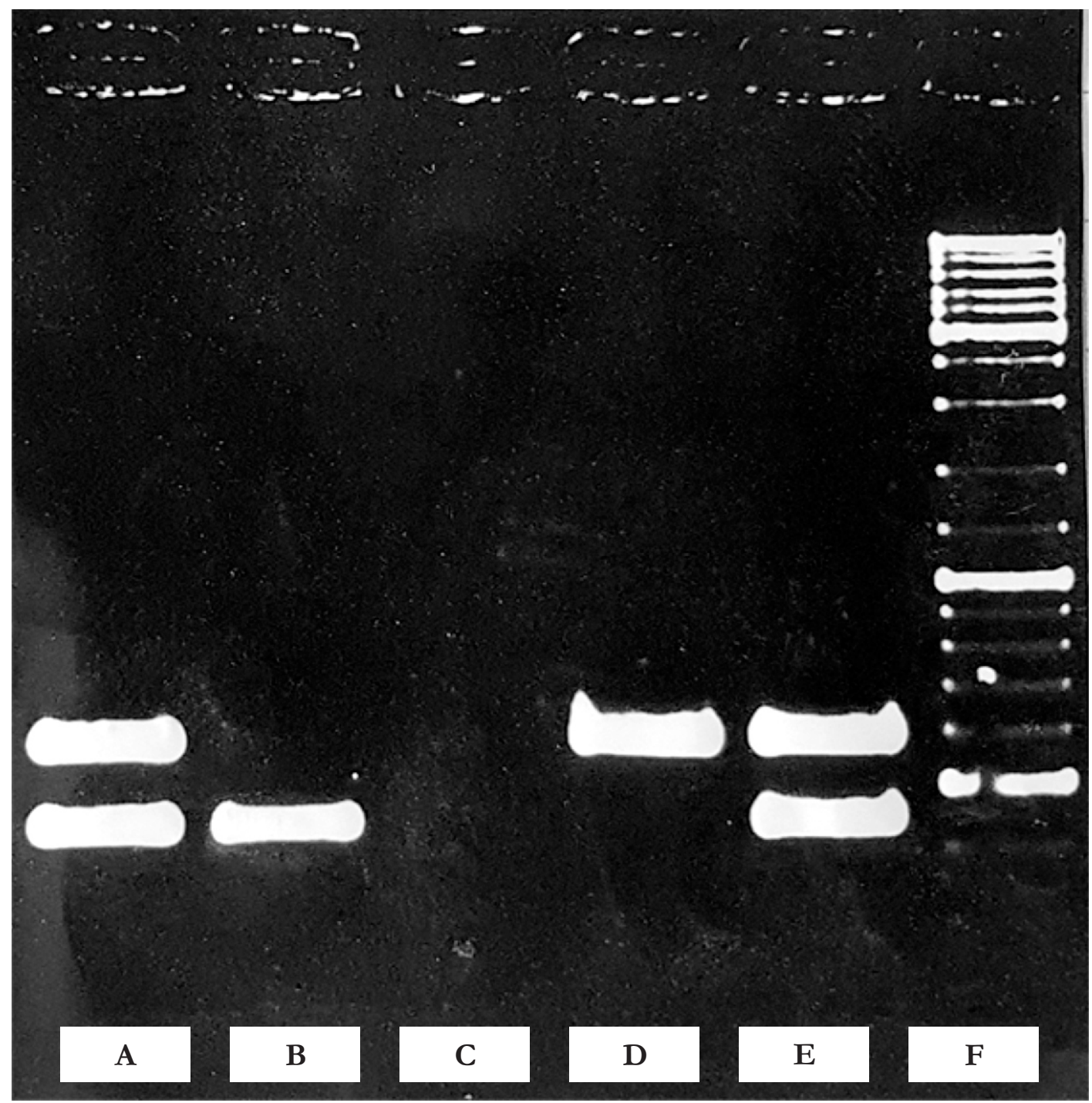

Figure 2: Electrophoretic Separation of the ESBL Genes

$\mathrm{A}=\left(b l a_{C T X-M} \& b l a_{T E M}\right), \mathrm{B}=b l a_{T E M}, \mathrm{C}=$ negative control, $\mathrm{D}=b l a_{C T X-M}, \mathrm{E}=\left(b l a_{C T X M} \& b l a_{T E M}\right)$ and $\mathrm{F}=$ ladder. $b l a_{\text {TEM }}=445 \mathrm{bp}, b l a_{C T X M}=593 \mathrm{bp}$.

Phylogroup A harboured co-existing bla $a_{C T X-M}$ and $b_{\text {TEM }}$ genes $2(66.7 \%)$ and one strain $(33.3 \%)$ harboured $b l a_{C T X M} ; 14(87.5 \%), 6(50 \%)$, and 5 $(83.3 \%)$ of isolates in the phylogroup clades I and II, group $\mathrm{E}$ and group F respectively harboured bla $a_{C T X: M}$ in combination with $b l a_{\text {TEM }}$ while the remaining isolates harboured only bla singly (as shown in figure1). The gel representative of the strains for ESBL is shown in figure 2.

\section{DISCUSSION}

The high mortality caused by the occurrence of ESBL- producing E. coli in clinical infections in our secondary and tertiary hospitals calls for the necessity to understand the genetic determinant and phylogroups of these isolates since the diversity in phylogroups is a marker for their virulence in extra intestinal infections and colonization. The presence of extendedspectrum beta-lactamase gene confers absolute resistance to the extended-spectrum cephalosporins making treatment options limited in infections caused by multi-drug resistant strains.

Phylogroup B2 is the most common in this study followed by Clade I and II and $\mathrm{E}$ with about $58 \%$, $14 \%$, and $11 \%$ respectively, majority of phylogroup B2 strains in our study were resistant to the tested third-generation cephalosporins and they harboured the ESBL genes of about 83\% $b l a_{\text {CTX-M }}$ and $b l a_{\text {TEM }}$ co-existing; $15.4 \% b l a_{C T X M}$ and 
$1.5 \% b l a_{T E M}$ but not $b l a_{S H V}$. Earlier reports of ESBL- producing isolates from clinical infections in Nigeria identified $b a_{C T X-M}$ as one of the important mechanisms that confer resistance to cephalosporins (Raji et al., 2015; Nwafia et al., 2019b) but most of the earlier studies did not categorize this resistance gene determinant into various phylogroups, which is a piece of important information for antimicrobial surveillance action in any community.

Only two strains in this study were classified as phylogroup $\mathrm{C}$, it is worthy to note that these two strains were $100 \%$ resistant to most of the antibiotics tested including amikacin but except for susceptibility to tigecycline and colistin. Strains belonging to phylogroup $\mathrm{C}$ appear to have a high level of antibiotic resistance and is consistent with a study in Iran where phylogroup $C$ was among the phylogroups in their study with multiple drug resistance but the genetic mechanisms that conferred resistance to the antibiotics including cephalosporins were not reported in their research (Iranpour et al., 2015), this indicates that phylogroup C are multi-resistant to cephalosporins and penicillins and the strains possess the gene responsible for the mechanisms of resistant to this antibiotics.

Phylogroup B2 was predominant among the isolates that were classified into phylogroups. Our study is consistent with other several studies (Kõljalg et al., 2014; Staji et al., 2019) where phylogroup B2 has been identified mostly in extraintestinal infections. This is because our isolates collections are from infection site and therefore belong to the pathogenic phylogroup B2. The B2 phylogroup strains are reported for its high virulence and multiple antibiotic resistance (Basu et al., 2013; Ranjan et al., 2017; Monroy-Pérez et al., 2020). This multiple antibiotic resistance was also observed in our study which predominantly harboured co-existing $b l a_{C T X-M}$ and $b l a_{T E M}$ gene, a similar study carried out in Iran also reported a high prevalence of $b l a_{\text {CTX-M }}$ among phylogroup B2 in their study (Nojoomi and Ghasemian, 2019). This is an indication of pathogenicity of phylogroup B2 strains in infections and the resistance to commonly used antibiotics in our hospitals, which can hinder management and treatment of infections caused by these strains.
Other phylogroups classified by Clermont such as phylogroups C, E, F, clades I and II were also identified in our collection of strains and harboured the bla $a_{C T X-M}$ gene. This confirms that isolates in these phylogroups are associated with infection and are isolated from extra-intestinal sites though only a few studies have been able to confirm the environmental and virulence characteristics of strains in this phylogroups.

Only $(3=2.7 \%)$ of the one hundred and thirteen isolates in this study belong to phylogroup A and only $(1=0.9 \%)$ belong to phylogroup B1. Strains belonging to phylogroup B1 and group $\mathrm{A}$ are classified and reported as commensals (Duriez et al., 2001; Chakraborty et al., 2015; Mosquito et al., 2015; Dadi et al., 2020) and these phylogroups are few in our isolate collection, this is probably because, the strains in this study were isolated from infection site and of pathogenic nature causing infection and not colonization or commensals as it was reported earlier that these phylogroups are predominant in gut flora and are commensals (Duriez et al., 2001; Chakraborty et al., 2015; Mosquito et al., 2015; Dadi et al., 2020). In addition B1 was identified from an animal, human and food product from another recent study (Olowe et al., 2019) and also the strains belonging to phylogroups B1 and A appeared to have fewer virulent determinant and therefore are commonly found to colonize a site as commensals and not a cause of infection.

About $5 \%$ of the isolates in this study belonged to phylogroup $\mathrm{F}$ which may be in line with the findings of Clermont et al. (2013) that this group is in close relationship with phylogroup B2 that made up the largest percentage of our isolates and therefore associated with infection.

About $7 \%$ of the isolates in this study could not be categorized. The reason for this is not clear but these may be newly emerging strains or novel strains whose phylotypes are yet to be discovered. Or the primers used could not bind to the DNA template, it may also be the variability in the genetic make-up of Escherichia coli which either gained or lost their gene(s) (mutated). About 5\% of ungroupable strain collection was also reported by Clermont et al. (2013). Multi-locus Sequence 
Typing (MLST), as well as Whole Genome Sequencing (WGS), are now a better option of grouping isolates into clonal groups especially when investigating the trend of transmission of particular isolates in a disease outbreak.

Our result confirms the high antibiotic-resistant nature of phylogroup B2 and their pathogenicity ability because they are strains that cause human infection. In addition, phylogroup class B2 harboured most of the different types of gene determinant or mechanism of extendedspectrum beta-lactam antibiotics resistance identified in this study such as co-existing $b l a_{C T X-M}$ and $b l a_{T E M}$ as well as bla $a_{C T X-M}$ occurring singly.

In conclusion, this study identified a high prevalence of the strains belonging to phylotype B2 among extra-intestinal clinical E. coli isolates from various hospitals in Lagos, Nigeria which harboured resistance genes to extended-spectrum cephalosporins. This information is important to drive national plans on strategies to curb and stop the spread of antibiotic resistance, especially among clinical infections in Nigerian hospital settings.

\section{ACKNOWLEDGEMENT}

We thank Prof Frieder Schaumburg of the Institute of Medical Microbiology, University Hospital Münster, Münster, Germany for providing the facilities to carry out the molecular techniques of the research and the DFG/TWAS for the cooperation visit scholarship received by AO to stay in Munster, Germany for this work.

\section{REFERENCES}

Adenipekun, E. O. Jackson, C. R. Ramadan, H. Iwalokun, B. A. Oyedeji, K. S. Frye, J. G. Barrett, J. B. Hiott, L. M. Woodley, T. A. Oluwadun, A. 2016. Prevalence and multidrug resistance of Escherichia coli from community-acquired infections in Lagos, Nigeria. Journal of Infection in Developing Countries, 10: 920-931.

Basu, S. Mukherjee, S. K. Hazra, A. Mukherjee, M. 2013. Molecular characterization of uropathogenic Escherichia coli: Nalidixic Acid and Ciprofloxacin resistance, virulent factors and phylogenetic background', Journal of Clinical and Diagnostic Research, 7:
2727-2731.

Chakraborty, A. Saralaya, V. Adhikari, P. Shenoy, S. Baliga, S. Hegde, A. 2015. Characterization of Escherichia coli phylogenetic groups associated with extra-intestinal infections in south Indian population. Annals of Medical and Health Sciences Research, 5: 241246.

Clermont, O. Christenson, J. K. Denamur, E. Gordon, D. M. 2013. The Clermont Escherichia coli phylo-typing method revisited: improvement of specificity and detection of new phylo-groups', EnvironmentalMicrobiology Reports, 5: 58-65.

Dadi, B. R. Abebe, T. Zhang, L. Mihret, A. Abebe, W. Amogne, W. 2020. Distribution of virulence genes and phylogenetics of uropathogenic Escherichia coli among urinary tract infection patients in Addis Ababa, Ethiopia', BMC Infectious Diseases, 20:108-116.

Duriez, P. Clermont, O. Bonacorsi, S. Bingen, E. Chaventré, A. Elion, J. Picard, B. Erick, D. 2001. Commensal Escherichia coli isolates are phylogenetically distributed among geographically distinct human populations', Microbiology, 147: 1671-1676.

Hertz, F. B. Jansåker, F. Okon, K. O. Abdulmumin, I. S. Onah, J. O. Ladan, J. Knudsen, J. D. 2019. ESBL-production in Escherichia coli and Klebsiella pneumoniae isolates from Nigeria', Microbiology open, 8: e00816.

Iranpour, D. Hassanpour, M. Ansari, H. Tajbakhsh, S. Khamisipour, G. Najafi, A. 2015. Phylogenetic groups of Escherichia coli strains from patients with urinary tract infection in Iran based on the new Clermont phylotyping method', Biomed Research International,: 846219 https://doi.org/10.1155/2015/846219

Kõljalg, S. Truusalu, K. Stsepetova, J. Pai, K. Vainumäe, I. Sepp, E. and Mikelsaar. 2014. 'The Escherichia coli phylogenetic group B2 with integrons prevails in childhood recurrent urinary tract infections', Acta Pathologica, Microbiologica, et Immunologica Scandinavica, 122: 452-458.

Lakoh, S., O. Adekanmbi, D. F. Jiba, G. F. Deen, W. Gashau, S. Sevalie, and E. Y. Klein. 2020. 'Antibiotic use among hospitalized adult patients in a setting with limited laboratory infrastructure in Freetown Sierra Leone, 
2017-2018', International Journal Infectious Diseases, 90: 71-76.

Monroy-Pérez, E. Cerón, A. B. García Cortés, L. R. Alonso, N. N. Domínguez-Trejo, P. Hernández-Jaimes, T. Bustos-Martínez, J. Hamdan-Partida, A. Rojas Jiménez, E. A. Vaca, S. Vaca-Paniagua, F. PaniaguaContreras 2020. 'Virulence gene transcription, phylogroups, and antibiotic resistance of cervico-vaginal pathogenic Escherichia. coli in Mexico', PloS one, 15: e0234730-e30.

Monstein, H. J., A. Ostholm-Balkhed, M. V. Nilsson, M. Nilsson, K. Dornbusch, and L. E. Nilsson. 2007. 'Multiplex PCR amplification assay for the detection of blaSHV, blaTEM and blaCTX-M genes in Enterobacteriaceae', Acta Pathologica, Microbiologica, et Immunologica Scandinavica, 115: 1400-1408.

Mosquito, S. Pons, M.J. Riveros, M. Ruiz, J. Ochoa, T. J. 2015. 'Diarrheagenic Escherichia coli Phylogroups are associated with antibiotic resistance and duration of diarrheal episode', The Scientific World Journal, 2015: 610403.

Munkhdelger, Y., N. Gunregjav, A. Dorjpurev, N. Juniichiro, and J. Sarantuya. 2017. 'Detection of virulence genes, phylogenetic group and antibiotic resistance of uropathogenic Escherichia coli in Mongolia', Journal of Infection in Developing Countries, 11: 51-57.

Nojoomi, F., and A. Ghasemian. 2019. "The relation of phylogroups, serogroups, virulence factors and resistance pattern of Escherichia coli isolated from children with septicemia." In New microbes and new infections, 100517.

Nwafia, I. N. Ohanu, M. E. Ebede, S. O. Ozumba, U. C. 2019a. 'Molecular detection and antibiotic resistance pattern of extendedspectrum beta-lactamase producing Escherichia coli in a Tertiary Hospital in Enugu, Nigeria', Annal of Clinical Microbioliology and Antimicrobial, 18: 41

Olalekan, A. Onwugamba, F. Iwalokun, B. Mellmann, A. Becker, K. Schaumburg, F. 2020 . 'High proportion of carbapenemase-producing Escherichia coli and Klebsiella pneumoniae among extendedspectrum $\beta$-lactamase-producers in
Nigerian hospitals', Journal of Global Antimicrobial Resistance, 21: 8-12.

Olowe, O. A. Adefioye, O. J. Ajayeoba, T. A. Schiebel, J. Weinreich, J Ali, A. Burdukiewicz, M. Rödiger, S. Schierack, P. 2019. 'Phylogenetic grouping and biofilm formation of multidrug resistant Escherichia coli isolates from humans, animals and food products in South-West Nigeria', Scientific African, 6: e00158.

Onanuga, A. Mahindroo, J. Singh, S. Taneja, N 2019. 'Phenotypic and molecular characterization of antimicrobial resistant Escherichia coli from urinary tract infections in Port-Harcourt, Nigeria', Pan Africa Medical Journal, 34:144.

Raji, M. A. Jamal, W. Ojemhen, O. Rotimi V. O. 2013. 'Point-surveillance of antibiotic resistance in Enterobacteriaceae isolates from patients in a Lagos Teaching Hospital, Nigeria', Journal of Infection and Public Health, 6: 431-437.

Raji, M. A. Jamal, W. Ojemeh, O. Rotimi, V. O. 2015. 'Sequence analysis of genes mediating extended-spectrum betalactamase (ESBL) production in isolates of Enterobacteriaceae in a Lagos Teaching Hospital, Nigeria', BMC Infectious Disease, 15: 259 .

Ranjan, A. Shaik, S. Nandanwar, N. Hussain, A. Tiwari, S. K. Semmler, T. Jadhav, S. Wieler, L. H. Alam, M. Colwell, R. R. Ahmed. N. 2017. 'Comparative genomics of Escherichia coli isolated from skin and soft tissue and other extraintestinal infections', mBio, 8:e01070-17.

Staji, H. Rassouli, M. Jourablou, S. 2019. 'Comparative virulotyping and phylogenomics of Escherichia coli isolates from urine samples of men and women suffering urinary tract infections', Iran Journal of Basic Medical Sciences, 22: 211-214.

Umeokonkwo, C. D. Madubueze, U. C. Onah, C. K. Okedo-Alex, I. N. Adeke, A. S. Versporten, A. Goossens, H. Igwe-Okomiso, D. Okeke, K. Azuogu, B. N. Onoh, R. 2019. 'Point prevalence survey of antimicrobial prescription in a tertiary hospital in South East Nigeria: A call for improved antibiotic stewardship', Journal of Global Antimicrobial Resistance, 17: 291-295. 